\title{
A LONG-LIVED ACCRETION DISK AROUND A LITHIUM-DEPLETED BINARY T TAURI STAR
}

\author{
Russel J. White and Lynne A. Hillenbrand \\ Department of Astronomy, California Institute of Technology, MS 105-24, Pasadena, CA 91125 \\ Received 2004 October 25; accepted 2005 January 12; published 2005 January 31
}

\begin{abstract}
We present a high-dispersion optical spectrum of St 34 and identify the system as a spectroscopic binary with components of similar luminosity and temperature (both $\mathrm{M} 3 \pm 0.5$ ). Based on kinematics, signatures of accretion, and location on an H-R diagram, we conclude that St 34 is a classical T Tauri star belonging to the TaurusAuriga T association. Surprisingly, however, neither component of the binary shows Li I $\lambda 6708$ absorption, the most universally accepted criterion for establishing stellar youth. In this uniquely known instance, the accretion disk appears to have survived longer than the lithium-depletion timescale. We speculate that the long-lived accretion disk is a consequence of the sub-AU separation companion tidally inhibiting, although not preventing, circumstellar accretion. Comparisons with pre-main-sequence evolutionary models imply, for each component of St 34, a mass of $0.37 \pm 0.08 M_{\odot}$ and an isochronal age of $8 \pm 3 \mathrm{Myr}$, which is much younger than the predicted lithium-depletion timescale of $\sim 25$ Myr. Although a distance $38 \%$ closer than that of Taurus-Auriga or a hotter temperature scale could reconcile this discrepancy at 21-25 Myr, similar discrepancies in other systems and the implications of an extremely old accreting Taurus-Auriga member suggest instead a possible problem with evolutionary models. Regardless, the older age implied by St 34's depleted lithium is the first compelling evidence of a substantial age spread in this region. Additionally, since St 34's coeval comembers with early M spectral types would likewise fail the lithium test for youth, current membership lists may be incomplete.
\end{abstract}

Subject headings: binaries: spectroscopic — stars: abundances — stars: pre-main-sequence

\section{INTRODUCTION}

$\mathrm{T}$ Tauri stars are a class of young ( $\$ 1-10 \mathrm{Myr})$ low-mass $\left(\sim 0.1-2 M_{\odot}\right)$ stars. Those that show signatures of accretion from a circumstellar disk, such as optical veiling and/or strong emission lines (e.g., $\mathrm{H} \alpha$ ), are called classical T Tauri stars, while those without accretion signatures are called weak-lined T Tauri stars. Observational studies of these stars have provided the foundation on which current theories of star and planet formation are based.

Initial efforts to find $\mathrm{T}$ Tauri stars primarily relied on objective-prism imaging surveys of dark clouds, in search of strong emission line stars (e.g., Joy 1949). This technique, while relatively effective, biased the discovered populations by mostly identifying classical T Tauri stars. Subsequent surveys that focused on proper motion (e.g., Jones \& Herbig 1979; Hartmann et al. 1991), infrared excesses (e.g., Kenyon et al. 1990), coronal/chromospheric indicators of youth, such as Xray emission (e.g., Walter et al. 1988; Wichmann et al. 1996), and $\mathrm{Ca}$ II $\mathrm{H}$ and $\mathrm{K}$ emission (e.g., Herbig et al. 1986), or location on an H-R diagram (e.g., Briceño et al. 1998), helped to establish a more complete and less biased census of star-forming regions (most notably Taurus-Auriga). However, since some older binary star systems (e.g., RS CVn-type stars) and postmain-sequence stars (e.g., asymptotic giant branch stars) also exhibit many of these same properties, confirmation of T Tauri status (i.e., extreme youth) has usually necessitated measurement of the surface abundance of ${ }^{7} \mathrm{Li}$. During the pre-mainsequence (PMS) contraction of a young star, ${ }^{7} \mathrm{Li}$ is destroyed via $(p, \alpha)$-reactions in the stellar interior when the central temperature rises above $\sim 3 \times 10^{6} \mathrm{~K}$ (Bodenheimer 1965). Because of rapid mixing in fully convective low-mass $\mathrm{T}$ Tauri stars, lithium is completely depleted in a small fraction of the contraction timescale (D'Antona \& Mazzitelli 1994; Bildsten et al. 1997; Baraffe et al. 1998; Burke et al. 2004). The presence of the strong, easily observable $\mathrm{Li}$ I $\lambda 6708$ absorption feature therefore implies that the star must be very young. The deple- tion timescale is a strong function of mass, however, being quickest ( $\sim 20 \mathrm{Myr}$ ) for stars of mass $\sim 0.6 M_{\odot}$. The onset of a radiative core, which inhibits efficient mixing, prior to full lithium depletion in higher mass stars delays their depletion timescale. The cooler central temperatures of lower mass stars likewise delay their depletion timescale; lithium-burning temperatures are never reached for substellar objects with $M \lesssim 0.06 M_{\odot}$. Thus, at the highest and lowest T Tauri star masses, other diagnostics are needed to confirm a star's extreme youth.

Here we present high-dispersion spectroscopic observations of St 34 (HBC 425; R.A. $=04^{\mathrm{h}} 54^{\mathrm{m}} 23.7$, Decl. $=+17^{\circ} 09^{\prime} 54^{\prime \prime}$, J2000.0; $V=14.4 \mathrm{mag}$ ), discovered as a strong $\mathrm{H} \alpha$ emission line star in the objective-prism survey of Stephenson (1986) and later shown to be an early to mid-M star (Downes \& Keyes 1988). Because of its strong $\mathrm{H} \alpha$ emission and location, it has been assumed to be a member of the Taurus-Auriga T association (Kenyon \& Hartmann 1995). Our new measurements demonstrate that St 34 is a spectroscopic binary and a classical $\mathrm{T}$ Tauri star, and we strengthen the case for its association with Taurus-Auriga. Unlike all other classical $\mathrm{T}$ Tauri stars and known members of Taurus-Auriga, however, St 34 has depleted its lithium. These results are used to assess the validity of evolutionary model predictions, the possibility of a lithiumdepleted population in Taurus-Auriga, and the influence of subAU separation companions on circumstellar disk lifetimes.

\section{SPECTROSCOPIC OBSERVATIONS AND INFERRED PROPERTIES}

The W. M. Keck I $10 \mathrm{~m}$ telescope and High Resolution Echelle Spectrometer (HIRES; Vogt et al. 1994) were used on 2003 February 17 to obtain a high-dispersion $(R \approx 34,000)$ optical spectrum (6330-8750 $\AA$ ) of St 34 . The observational setup, spectral calibration, and extraction are as described in White \& Hillenbrand (2004). Portions of the resulting spectrum of St 34 are shown in Figure 1. Its most distinguishing characteristics are strong, broad $\mathrm{H} \alpha$ emission, double-lined photo- 


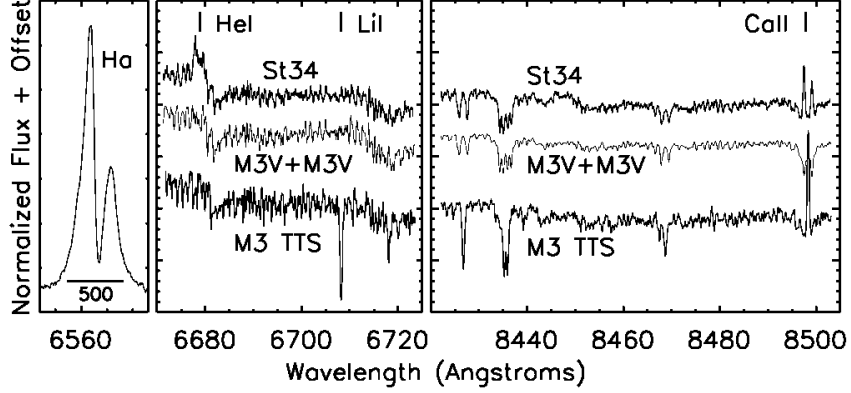

FIG. 1.-Portions of the Keck/HIRES spectrum of St 34 showing the strong, broad $\mathrm{H} \alpha$ emission profile (left panel) and two temperature-sensitive regions (middle and right panels); the wavelength scales are the same in all panels. The bar under the $\mathrm{H} \alpha$ profile indicates a velocity width of $500 \mathrm{~km} \mathrm{~s}^{-1}$. A synthetic spectroscopic binary, composed of two M3 dwarf stars, and the M3 weak-lined T Tauri star TWA 8a are also shown for comparison. St 34 shows no lithium in its spectrum.

spheric features (implying binarity), and no Li I $\lambda 6708$ absorption. A Li I equivalent width (EW) upper limit of $0.06 \AA$ for each spectroscopic component is determined by the size of features in the pseudocontinuum.

The stellar properties of St 34 are determined from an analysis of each component's spectral features. ${ }^{1}$ The procedure is described in White \& Hillenbrand (2004), but is tailored here for the analysis of a spectroscopic binary. Visual inspection of St 34's spectrum reveals that the two components are of similar brightness, well separated in velocity, and are slowly rotating. This allows a more independent analysis of each component. Radial velocities and $v \sin i$ values are measured by fitting the two peaks of the cross-correlation function determined using nonrotating early and mid-M dwarf templates. Each component's spectral type, system flux ratio, and continuum excess [or veiling, defined as $\left.r=F_{\text {excess }} /\left(F_{\text {prim }}+F_{\text {sec }}\right)\right]$ are determined simultaneously by comparisons with synthetic spectroscopic binaries generated by combining dwarf standards at the appropriate radial and rotational velocities. For the synthetic spectra, the spectral type of each component is allowed to vary from M0 to M5, the flux ratio from 0.5 to 2.0 , and the continuum excess from 0.0 to 2.0 . The best fit is determined by minimizing rms differences between St 34 and a synthetic binary spectrum over several temperature-sensitive regions (see White \& Hillenbrand 2004 for details). The components have the same spectral type (both $\mathrm{M} 3 \pm 0.5$ ) and are of similar brightness. The primary is assumed to be the star that is slightly $(10 \% \pm 4 \%)$ brighter at $6500 \AA$. Although the composite St 34 system appears to have a small amount of continuum excess at $6500 \AA\left(r_{6500}=0.13 \pm 0.05\right)$, it is not possible to tell if this excess is associated with only one or both of the components. No continuum excess is detected at longer wavelengths $\left(r_{8400}<0.09\right)$. Weak, narrow He I $\lambda 6678$ and $\mathrm{Ca}$ II $\lambda \lambda 8498$, 8662 emission is observed (Fig. 1), but not forbidden line emission (e.g., EW $\left[\mathrm{S}_{\text {II }}\right] \lambda \lambda 6716,6731<0.05 \AA$ ). Table 1 summarizes the spectroscopic properties of St 34 .

\section{EVIDENCE OF ADOLESCENCE}

The $\mathrm{H} \alpha$ emission line profile of St 34 is both strong (EW = $-51.6 \AA$ ) and broad (full width at $10 \%$ of the peak $=512$

\footnotetext{
${ }^{1}$ High spatial resolution imaging has identified a star at a distance of 1".2 from St 34, possibly making the system a hierarchical triple (R. White et al. 2004 , in preparation). This candidate companion is much fainter than the primary pair at $2.2 \mu \mathrm{m}(\Delta K=2.5 \mathrm{mag})$ and thus unlikely to contaminate the optical spectrum.
}

TABLE 1

Spectroscopic Properties of St 34

\begin{tabular}{|c|c|c|c|}
\hline Parameter & System & Primary & Secondary \\
\hline bs. Juliar & $2,452,68$ & .. & $\cdots$ \\
\hline x) $(\AA$ & -51.6 & $\ldots$ & .. \\
\hline$\alpha 10 \%$ width $\left(\mathrm{km} \mathrm{s}^{-1}\right)$ & 512 & & \\
\hline EW (Li I $)(\AA)$ & $\ldots$ & $<0.06$ & $<0.06$ \\
\hline si & & $<7.2$ & $<7.0$ \\
\hline Radial velocity ( & $17.9 \pm 0.6$ & $47.1 \pm 0.4$ & $-11.3 \pm 0.4$ \\
\hline Spectral type & & $\mathrm{M} 3 \pm 0.5$ & $\mathrm{M} 3 \pm 0.5$ \\
\hline$\left(F_{\text {prim }} / F_{\text {sec }}\right)_{6500} \ldots \ldots \ldots \ldots \ldots \ldots$ & $1.10 \pm 0.04$ & $\cdots$ & $\cdots$ \\
\hline$r_{6500} \ldots \ldots \ldots \ldots \ldots \ldots \ldots \ldots \ldots \ldots \ldots \ldots$ & $0.13 \pm 0.04$ & $\cdots$ & $\ldots$ \\
\hline$\left(F_{\text {prim }} / F_{\text {sec }}\right)_{8400} \ldots \ldots \ldots \ldots \ldots \ldots$ & $0.99 \pm 0.07$ & $\ldots$ & $\ldots$ \\
\hline$r_{8400} \ldots \ldots \ldots \ldots \ldots \ldots$ & $<0.09$ & & \\
\hline lass & $\ldots$ & $0.37 \pm 0.08$ & $0.37 \pm 0.08$ \\
\hline Isochronal age (Myr & $\ldots$ & $8 \pm 3$ & $8 \pm 3$ \\
\hline Li depletion age (Myr) & $\ldots$ & $>25$ & $>25$ \\
\hline
\end{tabular}

Note. - The systemic radial velocity is the average of the components. Its uncertainty does not reflect the uncertainties in their masses.

$\mathrm{km} \mathrm{s}^{-1}$ ). The breadth of this feature is not a consequence of binarity; the two peaks in the profile are separated in velocity by $\sim 180 \mathrm{~km} \mathrm{~s}^{-1}$, which is much greater than the velocity separation of the components $\left(58.4 \mathrm{~km} \mathrm{~s}^{-1}\right)$. Although some latetype main-sequence stars also display $\mathrm{H} \alpha$ emission caused by flares and other chromospheric activity, this emission is less intense and less broad than the profile of St 34 or any accreting young star (e.g., White \& Basri 2003). Chromospherically active main-sequence stars also tend to be rapidly rotating and $\mathrm{X}$-ray bright, in contrast to the small $v \sin i$ values and X-ray nondetection of St 34 (via ROSAT; König et al. 2001). Moreover, the observed strength of the $\mathrm{H} \alpha$ emission is similar to that seen in previous low spectral resolution observations of St 34 (EW $=-78 \AA$ A; Downes \& Keyes 1988; Kenyon \& Hartmann 1995), suggesting the current epoch was not a transient flarelike event. The $\mathrm{H} \alpha$ emission line profile of St 34, in light of its stellar properties, is most consistent with originating from a high-velocity accretion flow (e.g., Muzerolle et al. 2000). The low-level continuum excess at $6500 \AA$ supports the interpretation that one or both components of the binary are accreting, although the accretion rate is low $\left(2.5 \times 10^{-10} M_{\odot} \mathrm{yr}^{-1}\right.$, following a prescription similar to White $\&$ Hillenbrand 2004). Based on this evidence for accretion, we conclude that St 34 is a classical T Tauri star.

The reservoir of accreting material has not yet been detected, however. St 34 has no measurable near-infrared $\left(K_{s}\right)$ excess, based on comparing the observed $K_{s}$ magnitude to that predicted from the spectral type, extinction (determined below), and $J$ magnitude. Although Weaver \& Jones (1992) claim St 34 was detected by IRAS at 12, 25, and $60 \mu \mathrm{m}$, inspection of the IRAS Sky Survey Atlas, even after the more up-to-date HiRes processing, reveals no point or extended source within several arcminutes of St 34 . We suggest the Weaver \& Jones (1992) identification was spurious. St 34 was also not detected in the $1.3 \mathrm{~mm}$ survey by Osterloh \& Beckwith (1995), $F_{1.3 \mathrm{~mm}}<15 \mathrm{mJy}$, which is sensitive to cool outer-disk material. We emphasize, however, that the lack of detected excess emission is consistent with St 34's low-level accretion and cool stellar temperature; several low-mass accreting stars in TaurusAuriga have not yet been detected at far-infrared and millimeter wavelengths either (Kenyon \& Hartmann 1995).

Kinematic information supports the assertion that St 34 is a member of the Taurus-Auriga $\mathrm{T}$ association. Assuming that the spectroscopic binary components have the same mass, as suggested by their similar spectral type and brightness, the systemic radial velocity is $17.9 \pm 0.6 \mathrm{~km} \mathrm{~s}^{-1}$, which is identical to the 
mean of Taurus members (17.8 $\mathrm{km} \mathrm{s}^{-1}$; Hartmann et al. 1986).

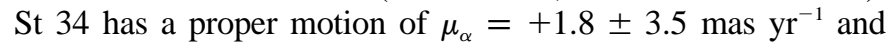
$\mu_{\delta}=-12.6 \pm 3.5$ mas $\mathrm{yr}^{-1}$, which, in combination with its radial velocity and an assumed Taurus distance of $145 \pm 10 \mathrm{pc}$ (Bertout et al. 1999), corresponds to space motion of $U=$ $-15.6 \pm 1.5, V=-8.4 \pm 2.2$, and $W=-8.8 \pm 2.3 \mathrm{~km} \mathrm{~s}^{-1}$ (E. Mamajek 2004, private communication). This motion is statistically most consistent with that of lithium-rich Taurus members (e.g., Jones \& Herbig 1979), as opposed to nearby moving groups (e.g., $\beta$ Pictoris; Zuckerman \& Song 2004). Thus, based on evidence for extreme youth, spatial proximity (within 1.5 of L1558), and space motion, we conclude that St 34 is a member of the Taurus-Auriga T association.

Mass and age estimates for St 34 are determined by comparing the temperature and luminosity with the Baraffe et al. (1998) PMS evolutionary models. A temperature of $3415 \mathrm{~K}$ is assigned using the spectral type-temperature scale of Luhman et al. (2003), which is slightly hotter than a typical dwarf temperature scale, but yields coeval cluster populations in combination with this evolutionary model. A visual extinction of 0.24 mag is determined by comparing the $J-H$ color to that expected for an M3 star (Kirkpatrick \& McCarthy 1994), using a standard interstellar extinction law (Rieke \& Lebofsky 1985). The Two Micron All Sky Survey magnitudes of St 34 are $J=$ $10.69 \pm 0.02, H=10.08 \pm 0.02$, and $K_{s}=9.79 \pm 0.02$; no reliable optical colors are available. Luminosity is computed by applying a bolometric correction of +1.75 to the reddeningcorrected $J$ magnitude, which is split assuming equal contribution from each component of the binary. A distance of $145 \pm 10 \mathrm{pc}$ yields $\log \left(L / L_{\odot}\right)=-1.03 \pm 0.06$ for each component. In the top panel of Figure 2, St 34 is shown on an H-R diagram, along with the Baraffe et al. (1998) evolutionary models. For each component, the implied age is $8 \pm 3 \mathrm{Myr}$, and mass is $0.37 \pm 0.08 M_{\odot}$. St 34 appears to be somewhat older than most stars in Taurus, which have an average age of 2-3 Myr (White \& Ghez 2001), and are all thought to be younger than $4 \mathrm{Myr}$ (Hartmann 2003).

In the bottom panel of Figure 2, the lithium abundance of St 34 is compared to the lithium-depletion predictions of the same Baraffe et al. (1998) evolutionary models. The Li $\lambda 6708$ equivalent width of $\lesssim 0.06 \AA$ corresponds to a lithium abundance of $\log n(\mathrm{Li}) \lesssim 0.3$ dex, following the curves of growth shown in Song et al. (2002; Fig. 2). This amount of depletion implies an age $\gtrsim 25$ Myr. Comparisons with other evolutionary models yield lithium-depletion ages that agree to within $20 \%$ (see, e.g., Burke et al. 2004).

\section{DISCUSSION AND IMPLICATIONS}

\subsection{A Possible Problem for Lithium-depletion Predictions}

Although the isochronal age inferred for St 34 is much younger than its lithium-depletion age, a distance of $90 \mathrm{pc}$ instead of the assumed $145 \mathrm{pc}$ would increase the isochronal age to $25 \mathrm{Myr}$, consistent with the lithium-depletion age. Similarly, if the assumed temperature is increased to $3600 \mathrm{~K}$ (which is $340 \mathrm{~K}$ hotter than a typical M3 dwarf temperature), the isochrone and lithium-depletion ages agree at $21 \mathrm{Myr}$. At an age $\gtrsim 20$ Myr, however, St 34 would be by far the oldest classical T Tauri star known; MP Mus in Scorpius-Centaurus (age $\sim 13$ Myr; Mamajek et al. 2002) is currently thought to be one of the oldest. St 34 would also be older than typical cloud dispersal timescales (<10-20 Myr; Palla \& Galli 1997), which would call into question its association with Taurus-Auriga, as location and kinematics suggest. This large age for St 34 seems unlikely. Song et al. (2002) identified a similar dis-

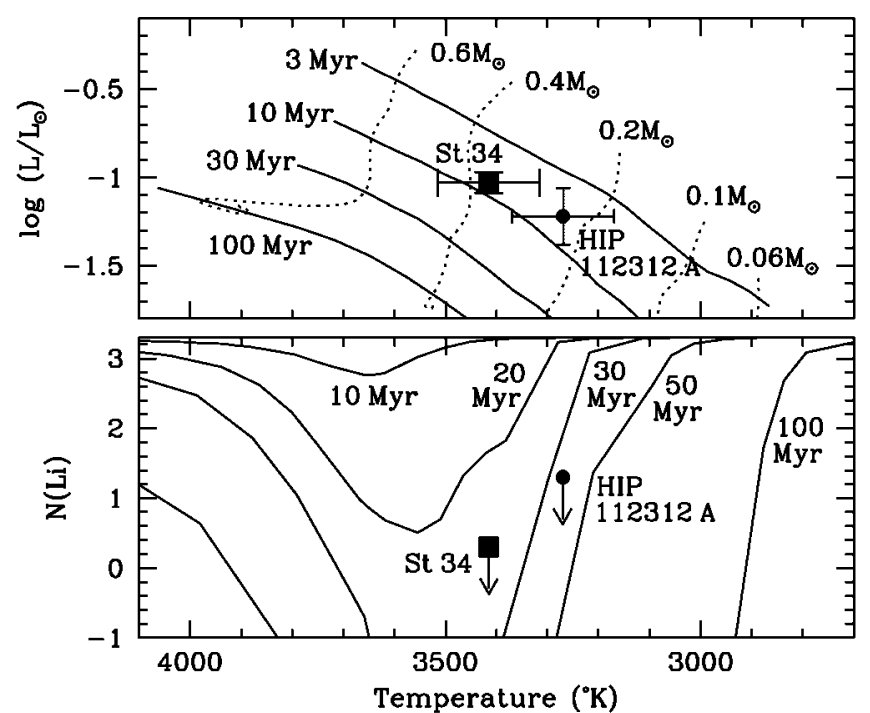

FIG. 2.-Top: St 34 (square) and HIP 112312 A (circle) shown on an H-R diagram, along with the evolutionary models of Baraffe et al. (1998). Since both components of St 34 have the same temperature and luminosity, only one point is visible. Bottom: St 34 and HIP 112312 A are shown on a lithium abundance vs. temperature diagram, along with the isochrones predicted by the same Baraffe et al. (1998) models. Although the location of St 34 and HIP $112312 \mathrm{~A}$ on the H-R diagram suggests ages $\lesssim 10$ million years, the depleted lithium abundance of both stars suggests ages older than $\sim 25$ and $\sim 35 \mathrm{Myr}$, respectively.

crepancy between isochronal and lithium-depletion ages in the case of HIP 112312 A. The low lithium abundance of this M4 star implies an age $\gtrsim 35 \mathrm{Myr}$, while its isochronal age, which is based on a Hipparcos-determined distance, is $6 \pm 3 \mathrm{Myr}$ (Fig. 2). As with St 34, a hotter temperature could reconcile these ages at 20-25 Myr. Finally, we note that the lithium-depletion ages of young open clusters, including the Pleiades, $\alpha$ Persei, IC 2391, and NGC 2547 (Burke et al. 2004), are all systematically larger than the isochronal ages fitted to both low-mass unevolved members and the upper main sequence near the nuclear turnoff (Stauffer et al. 2001; Jeffries \& Naylor 2001). Overall, the emerging observational evidence suggests a problem with lithium-depletion ages, being systematically too old, although a problem with the PMS temperature scale, being too cool, cannot be ruled out.

\subsection{Implications for the Taurus Population}

If St 34 is a member of the Taurus-Auriga $\mathrm{T}$ association, as evidence suggests, it presents the first strong case for a significant age spread in this region. St 34 must be older than currently forming stars by an amount equal to the lithiumdepletion timescale. Evolutionary models suggest this could be as large as 21-25 Myr, but as noted above, these values appear to be extreme. The age spread would be more accurately constrained if additional older, possibly lithium-depleted, members are discovered. St 34 was easily identified because of its strong $\mathrm{H} \alpha$ emission. Lithium-depleted weak-lined T Tauri stars, on the other hand, even if identified and observed spectroscopically, would have been dismissed thus far as nonmembers. In order to estimate how many older weak-lined systems there could be in Taurus, we use the ratio of weak-lined to classical T Tauri stars in clusters of age $\sim 10 \mathrm{Myr}$, which range from $\sim 90 \%$ for the TW Hydrae association (age 8 Myr; Zuckerman \& Song 2004) to $\sim 99 \%$ for the Sco-Cen subgroups (age $~ 13$ Myr; Mamajek et al. 2002). If surveys of these clusters are also biased by requiring the presence of lithium absorption for a member to be 
confirmed, these ratios could be even higher. This suggests that St 34 may have 10 and possibly many tens of coeval comembers that are weak-lined $\mathrm{T}$ Tauri stars. Those of early $\mathrm{M}$ spectral type will likely have depleted their lithium (Fig. 2). Current membership lists of Taurus may therefore be incomplete.

The Einstein and ROSAT surveys may have identified some of these lithium-poor stars. Walter et al. (1988) and Wichmann et al. (1996) together identified 17 stars with spectral types M1-M3.5 in Taurus, nearly half of which have been subsequently dismissed as nonmembers because of depleted lithium. Some of these lithium-poor stars nevertheless have radial velocities consistent with Taurus (e.g., RX J0446.8+2255; Wichmann et al. 2000), suggesting they could in fact be bona fide members. Confirmation of membership will require more accurate estimates of distance, proper motion, and surface gravity. We note that if this proposed older population is identified, it has significant implications for the duration of star formation in Taurus, which is generally believed to be less than $4 \mathrm{Myr}$ (Hartmann 2003; but see Palla \& Stahler 2002), and for the initial mass function of Taurus, which apparently peaks at a mass $\left(\sim 0.8 M_{\odot}\right.$; Luhman et al. 2003), slightly larger than the mass at which lithium depletion occurs first.

\subsection{Long-lived Accretion Disks in Close Binary Systems}

With an age of $\gtrsim 8 \mathrm{Myr}$, St 34 is one of a handful of old ( $\gtrsim 10$ Myr) classical T Tauri stars. We speculate than in many cases, these long-lived accretion disks are a consequence of a tidally inhibited accretion flow caused by a sub-AU separation companion. The masses and relative velocities of the components of St 34, for example, imply a binary separation of $\lesssim 0.78$ AU. As has been identified in some spectroscopic binary systems (e.g., DQ Tau; Basri et al. 1997) and predicted by numerical simulations (Artymowicz \& Lubow 1996), the orbital dynamics of a close binary do not preclude accretion from a circumbinary disk. Nevertheless, we suggest that it is less efficient. In support of this, there is some evidence for a higher frequency of spectroscopic binaries among old classical $\mathrm{T}$ Tauri stars than among younger $\mathrm{T}$ Tauri populations, for which the binary fraction is only $7 \% \pm 3 \%$ (Mathieu 1994; for periods less than 100 days). Of the four classical T Tauri stars in the TW Hydrae association, 50\% are spectroscopic binaries (TWA 5A, Hen 3-600A; Muzerolle et al. 2000; Mohanty et al. 2003). Of the remaining two accreting stars (TW Hya, TWA 14), only TW Hya has multiple high-dispersion measurements sensitive to radial velocity variations, but its pole-on orientation (Weinberger et al. 2002) would significantly inhibit the detection of a close companion in a coplanar orbit. Both TWA 14 and TW Hya could be yet unidentified sub-AU binary systems. A more complete binary census of these and other old accretors (e.g., MP Mus; Mamajek et al. 2002) is needed to confirm this hypothesis. One interesting implication is that planets would have a longer time to form in the circumbinary disk of sub-AU separation binary stars than around single stars.

We thank I. Baraffe, A. Ghez, and J. Stauffer for helpful discussions, and are grateful to E. Mamajek for generously providing valuable kinematic information and insight. We appreciate the data provided by the NASA/IPAC Infrared Science Archive, and the privilege to observe on the revered summit of Mauna Kea.

\section{REFERENCES}

Artymowicz, P., \& Lubow, S. H. 1996, ApJ, 467, L77

Baraffe, I., Chabrier, G., Allard, F., \& Hauschildt, P. H. 1998, A\&A, 337, 403

Basri, G., Johns-Krull, C. M., \& Mathieu, R. D. 1997, AJ, 114, 781

Bertout, C., Robichon, N., \& Arenou, F. 1999, A\&A, 352, 574

Bildsten, L., Brown, E. F., Matzner, C. D., \& Ushomirsky, G. 1997, ApJ, 482, 442

Bodenheimer, P. 1965, ApJ, 142, 451

Briceño, C., Hartmann, L., Stauffer, J., \& Martín, E. 1998, AJ, 115, 2074

Burke, C. J., Pinsonnealult, M. H., \& Sills, A. 2004, ApJ, 604, 272

D’Antona, F., \& Mazzitelli, I. 1994, ApJS, 90, 467

Downes, R. A., \& Keyes, C. D. 1988, AJ, 96, 777

Hartmann, L. 2003, ApJ, 585, 398

Hartmann, L., Hewett, R., Stahler, S., \& Mathieu, R. D., 1986, ApJ, 309, 275

Hartmann, L., Stauffer, J. R., Kenyon, S. J., \& Jones, B. F. 1991, AJ, 101, 1050

Herbig, G. H., Vrba, F. J., \& Rydgren, A. E. 1986, AJ, 91, 575

Jeffries, R. D., \& Naylor, T. 2001, in ASP Conf. Ser. 243, From Darkness to Light: Origin and Evolution of Young Stellar Clusters, ed. T. Montmerle \& P. André (San Francisco: ASP), 633

Jones, B. F., \& Herbig, G. H. 1979, AJ, 84, 1872

Joy, A. H. 1949, ApJ, 110, 424

Kenyon, S. J., \& Hartmann, L. W. 1995, ApJS, 101, 117

Kenyon, S. J., Hartmann, L. W., Strom, K. M., \& Strom, S. E. 1990, AJ, 99, 869

Kirkpatrick, J. D., \& McCarthy, D. W., Jr. 1994, AJ, 107, 333

König, B., Neuhäuser, R., \& Stelzer, B. 2001, A\&A, 369, 971

Luhman, K. L., Briceño, C., Stauffer, J. R., Hartmann, L., Barrado y Navascués, D., \& Caldwell, N. 2003, ApJ, 590, 348
Mamajek, E. E., Meyer, M. R., \& Liebert, J. 2002, AJ, 124, 1670

Mathieu, R. D. 1994, ARA\&A, 32, 465

Mohanty, S., Jayawardhana, R., \& Barrado y Navascués, D. 2003, ApJ, 593, L109

Muzerolle, J., Calvet, N., Briceño, C., Hartmann, L., \& Hillenbrand, L. 2000, ApJ, 535, L47

Osterloh, M., \& Beckwith, S. V. W. 1995, ApJ, 439, 288

Palla, F., \& Galli, D. 1997, ApJ, 476, L35

Palla, F., \& Stahler, S. W. 2002, ApJ, 581, 1194

Rieke, G. H., \& Lebofsky, M. J. 1985, ApJ, 288, 618

Song, I., Bessell, M. S., \& Zuckerman, B. 2002, ApJ, 581, L43

Stauffer, J. R., Jeffries, R. D., Martín, E. L., \& Turndrup, D. M. 2001, in ASP Conf. Ser. 223, Cool Stars, Stellar Systems and the Sun, Ed. R. J. García López, R. Rebolo, \& M. Zapatero Osorio (San Francisco: ASP), 399

Stephenson, C. B. 1986, ApJ, 300, 779

Vogt, S. S., et al. 1994, Proc. SPIE, 2198, 362

Walter, F. M., Brown, A., Mathieu, R. D., Myers, P. C., \& Vrba, F. J. 1988, AJ, 96, 297

Weaver, W. B., \& Jones, G. 1992, ApJS, 78, 239

Weinberger, A. J., et al. 2002, ApJ, 566, 409

White, R. J., \& Basri, G. 2003, ApJ, 582, 1109

White, R. J., \& Ghez, A. M. 2001, ApJ, 556, 265

White, R. J., \& Hillenbrand, L. A. 2004, ApJ, 616, 998

Wichmann, R. et al. 1996, A\&A, 312, 439

. 2000, A\&A, 359, 181

Zuckerman, B., \& Song, I. 2004, ARA\&A, 42, 685 